\title{
New versions of the Hestenes-Stiefel nonlinear conjugate gradient method based on the secant condition for optimization
}

\author{
LI ZHANG \\ College of Mathematics and Computational Science \\ Changsha University of Science and Technology \\ Changsha 410076, China \\ E-mail: mathlizhang@126.com
}

\begin{abstract}
Based on the secant condition often satisfied by quasi-Newton methods, two new versions of the Hestenes-Stiefel (HS) nonlinear conjugate gradient method are proposed, which are descent methods even with inexact line searches. The search directions of the proposed methods have the form $d_{k}=-\theta_{k} g_{k}+\beta_{k}^{H S} d_{k-1}$, or $d_{k}=-g_{k}+\beta_{k}^{H S} d_{k-1}+\theta_{k} y_{k-1}$. When exact line searches are used, the proposed methods reduce to the standard HS method. Convergence properties of the proposed methods are discussed. These results are also extended to some other conjugate gradient methods such as the Polak-Ribiére-Polyak (PRP) method. Numerical results are reported.
\end{abstract}

Mathematical subject classification: $90 \mathrm{C} 30,65 \mathrm{~K} 05$.

Key words: HS method, descent direction, global convergence.

\section{Introduction}

Assume that $f: R^{n} \rightarrow R$ is a continuously differentiable function whose gradient is denoted by $g$. The problem considered in this paper is

$$
\min f(x), \quad x \in R^{n} .
$$

The iterates for solving (1.1) are given by

$$
x_{k+1}=x_{k}+\alpha_{k} d_{k},
$$

\#CAM-53/09. Received: 08/II/09. Accepted: 15/II/09. 
where the stepsize $\alpha_{k}$ is positive and computed by some line search, and $d_{k}$ is the search direction.

Conjugate gradient methods are very efficient iterative methods for solving (1.1) especially when $n$ is large. The search direction has the following form:

$$
d_{k}= \begin{cases}-g_{k}, & \text { if } k=0, \\ -g_{k}+\beta_{k} d_{k-1}, & \text { if } k>0,\end{cases}
$$

where $\beta_{k}$ is a parameter. Some well-known conjugate gradient methods include the Polak-Ribiére-Polyak (PRP) method [17, 18], the Hestenes-Stiefel (HS) method [13], the Liu-Storey (LS) method [15], the Fletcher-Reeves (FR) method [8], the Dai-Yuan (DY) method [5] and the conjugate descent (CD) method [9]. In this paper, we are interested in the HS method, in which $\beta_{k}$ is defined by

$$
\beta_{k}^{H S}=\frac{g_{k}^{T} y_{k-1}}{d_{k-1}^{T} y_{k-1}},
$$

where $y_{k-1}=g_{k}-g_{k-1}$. Throughout the paper, we denote $s_{k-1}=x_{k}-x_{k-1}=$ $\alpha_{k-1} d_{k-1}$, and $\|\cdot\|$ stands for the Euclidean norm. Convergence properties of conjugate gradient methods can be found in the book [6], the survey paper [12] and references therein.

The HS method behaves like the PRP method in practical computation and is generally regarded as one of the most efficient conjugate gradient methods. An important feature of the HS method is that it satisfies conjugacy condition

$$
d_{k}^{T} y_{k-1}=0
$$

which is independent of the objective function and line search. However, Dai and Liao [4] pointed out that in the case $g_{k+1}^{T} d_{k} \neq 0$, the conjugacy condition (1.5) may have some disadvantages (for instance, see [21]). In order to construct a better formula for $\beta_{k}$, Dai and Liao proposed a new conjugacy condition and a new conjugate gradient method called the Dai-Liao (DL) method, given by

$$
\beta_{k}^{D L}=\frac{g_{k}^{T}\left(y_{k-1}-t s_{k-1}\right)}{d_{k-1}^{T} y_{k-1}}
$$

with a parameter $t \in[0, \infty)$. Based on the idea of the DL method, Hager and Zhang [11] proposed a descent conjugate gradient method (HZ). 
Besides conjugate gradient methods, the following gradient type methods

$$
d_{k}= \begin{cases}-g_{k}, & \text { if } k=0, \\ -\theta_{k} g_{k}+\beta_{k} d_{k-1}, & \text { if } k>0,\end{cases}
$$

have also been studied extensively by many authors. Here $\theta_{k}$ and $\beta_{k}$ are two parameters. Clearly, if $\theta_{k}=1$, the methods (1.7) become conjugate gradient methods (1.3). When

$$
\beta_{k}=\frac{\left(\theta_{k} y_{k-1}-s_{k-1}\right)^{T} g_{k}}{d_{k-1}^{T} y_{k-1}},
$$

the methods (1.7) reduce to spectral conjugate gradient methods [2] and scaled conjugate gradient methods [1]. Yuan and Stoer [21] proposed a subspace method to compute the parameters $\theta_{k}$ and $\beta_{k}$ which solve the following subproblem

$$
\min _{d \in \Omega_{k}} \phi_{k}(d)=g_{k}^{T} d+\frac{1}{2} d^{T} B_{k} d,
$$

where $\Omega_{k}=\operatorname{Span}\left\{g_{k}, d_{k-1}\right\}$ and $B_{k}$ is a suitable quasi-Newton matrix such as the memoryless BFGS update matrix [19]. Zhang et al. [23] proposed a modified FR method where the parameters in (1.7) are given by

$$
\theta_{k}=\frac{d_{k-1}^{T} y_{k-1}}{\left\|g_{k-1}\right\|^{2}}, \quad \beta_{k}=\beta_{k}^{F R}=\frac{\left\|g_{k}\right\|^{2}}{\left\|g_{k-1}\right\|^{2}} .
$$

This method satisfies $g_{k}^{T} d_{k}=-\left\|g_{k}\right\|^{2}$ and this property depends neither on the line search used, nor on the convexity of the objective function. Moreover, this method converges globally for nonconvex functions with Armijo or Wolfe line search.

Recently, based on the direction generated by the memoryless BFGS update matrix [19], Zhang et al. [22, 24] proposed the following three-term conjugate gradient type method,

$$
d_{k}= \begin{cases}-g_{k}, & \text { if } k=0, \\ -g_{k}+\beta_{k} d_{k-1}+\theta_{k} y_{k-1}, & \text { if } k>0,\end{cases}
$$

where $\theta_{k}$ and $\beta_{k}$ are two parameters. If the parameters in (1.8) are given by

$$
\beta_{k}=\beta_{k}^{P R P}=\frac{g_{k}^{T} y_{k-1}}{\left\|g_{k-1}\right\|^{2}}, \quad \theta_{k}=-\frac{g_{k}^{T} d_{k-1}}{\left\|g_{k-1}\right\|^{2}},
$$


then this method becomes the modified PRP method [22]. If the parameters in (1.8) are chosen as

$$
\beta_{k}=\beta_{k}^{H S}, \quad \theta_{k}=-\frac{g_{k}^{T} d_{k-1}}{d_{k}^{T} y_{k-1}},
$$

then we get the three-term HS method [24]. Both methods still retain the relation $g_{k}^{T} d_{k}=-\left\|g_{k}\right\|^{2}$ and performed well in practical computations.

In this paper, we are concerned with the methods (1.7) and (1.8) with the parameter $\beta_{k}=\beta_{k}^{H S}$. Then we try to construct new $\theta_{k}$ by using idea of the DL method [4].

This paper is organized as follows. In Section 2, we present new formulas for $\theta_{k}$ and corresponding algorithms. In Section 3, we analyze global convergence properties of the proposed methods with some inexact line searches. In Section 4, we extend the results of Section 2 and Section 3 to other conjugate gradient methods. In Section 5, we report numerical comparisons with existing conjugate gradient methods by using problems in the CUTE library [3].

\section{New formula for $\theta_{k}$ and algorithms}

In this section, we first describe the following two-terms HS conjugate gradient type method,

$$
d_{k}= \begin{cases}-g_{k}, & \text { if } k=0, \\ -\left(1+\theta_{k}^{\prime}\right) g_{k}+\beta_{k}^{H S} d_{k-1}, & \text { if } k>0\end{cases}
$$

where, for convenience, we write $\theta_{k}=1+\theta_{k}^{\prime}$.

In order to introduce our method, let us simply recall the conjugacy condition proposed by Dai and Liao [4]. Linear conjugate gradient methods generate a search direction such that the conjugacy condition holds, namely,

$$
d_{i}^{T} Q d_{j}=0 \quad \forall i \neq j
$$

where $Q$ is the symmetric and positive definite Hessian matrix of the quadratic objective function $f(x)$. For general nonlinear functions, it follows from the mean value theorem that there exists some $\tau \in(0,1)$ such that

$$
d_{k}^{T} y_{k-1}=\alpha_{k-1} d_{k}^{T} \nabla^{2} f\left(x_{k-1}+\tau \alpha_{k-1} d_{k-1}\right) d_{k-1} .
$$


Therefore it is reasonable to replace (2.2) by the following conjugacy condition:

$$
d_{k}^{T} y_{k-1}=0 .
$$

Dai and Liao [4] used the secant condition of quasi-Newton methods, that is,

$$
H_{k} y_{k-1}=s_{k-1},
$$

where $H_{k}$ is an approximation to the inverse Hessian. For quasi-Newton methods, the search direction $d_{k}$ can be calculated in the form

$$
d_{k}=-H_{k} g_{k}
$$

By the use of (2.4) and (2.5), we get that

$$
d_{k}^{T} y_{k-1}=-\left(H_{k} g_{k}\right)^{T} y_{k-1}=-g_{k}^{T}\left(H_{k} y_{k-1}\right)=-g_{k}^{T} s_{k-1} .
$$

The above relation implies that (2.3) holds if the line search is exact since in this case $g_{k}^{T} s_{k-1}=0$. However, practical numerical algorithms normally adopt inexact line searches instead of exact ones. For this reason, Dai and Liao replaced the above conjugacy condition by

$$
d_{k}^{T} y_{k-1}=-\operatorname{tg}_{k}^{T} s_{k-1},
$$

where $t$ is a scalar. If we substitute (1.3) into (2.6), we get the formula for $\beta_{k}^{D L}$ in (1.6).

In order to get the formula for $\theta_{k}$ in our method, substituting (2.1) into (2.6), we have

$$
\theta_{k}^{\prime} g_{k}^{T} y_{k-1}=\operatorname{tg}_{k}^{T} s_{k-1}
$$

Set

$$
t=h g_{k}^{T} y_{k-1},
$$

where $h$ is a parameter. We get from the above two equalities that

$$
\theta_{k}^{\prime}=h g_{k}^{T} s_{k-1} .
$$

Now let

$$
h=\frac{g_{k}^{T} y_{k-1}}{s_{k-1}^{T} y_{k-1}\left\|g_{k}\right\|^{2}}-\frac{\rho}{s_{k-1}^{T} y_{k-1}},
$$


with $\rho \in[0,1]$. Then, we have

$$
\theta_{k}^{\prime}=\beta_{k}^{H S} \frac{g_{k}^{T} d_{k-1}}{\left\|g_{k}\right\|^{2}}-\rho \frac{g_{k}^{T} d_{k-1}}{d_{k-1}^{T} y_{k-1}} .
$$

For convenience, we summarize the above method as the following algorithm which we call the two-term HS method.

Algorithm 2.1 (two-term HS Method):

Step 0: Given the constant $\rho \in[0,1]$, choose an initial point $x_{0} \in R^{n}$. Let $k:=0$.

Step 1: Compute $d_{k}$ by

$$
d_{k}= \begin{cases}-g_{k}, & \text { if } k=0, \\ -\theta_{k} g_{k}+\beta_{k}^{H S} d_{k-1}, & \text { if } k>0,\end{cases}
$$

where

$$
\theta_{k}=1+\theta_{k}^{\prime}=1+\beta_{k}^{H S} \frac{g_{k}^{T} d_{k-1}}{\left\|g_{k}\right\|^{2}}-\rho \frac{g_{k}^{T} d_{k-1}}{d_{k-1}^{T} y_{k-1}} .
$$

Step 2: Determine $\alpha_{k}$ by some line search.

Step 3: Let the next iterate be $x_{k+1}=x_{k}+\alpha_{k} d_{k}$.

Step 4: Let $k:=k+1$. Go to Step 1 .

By the same argument as Algorithm 2.1, we can get the following three-term HS method. In the rest of this paper, we only give the direction in the algorithm where the other steps are as same as Algorithm 2.1 since conjugate gradient methods are mainly determined by their search directions.

Algorithm 2.2 (three-term HS Method):

$$
d_{k}= \begin{cases}-g_{k}, & \text { if } k=0, \\ -g_{k}+\beta_{k}^{H S} d_{k-1}+\theta_{k}^{(1)} y_{k-1}, & \text { if } k>0,\end{cases}
$$

where

$$
\theta_{k}^{(1)}=\rho \frac{\left\|g_{k}\right\|^{2}}{g_{k}^{T} y_{k-1}} \frac{g_{k}^{T} d_{k-1}}{d_{k-1}^{T} y_{k-1}}-\frac{g_{k}^{T} d_{k-1}}{d_{k-1}^{T} y_{k-1}} .
$$


Remark 2.1. It is interesting to note that when $\rho=0$ in (2.7)-(2.8) or (2.9)-(2.10), we have

$$
d_{k}^{T} g_{k}=-\left\|g_{k}\right\|^{2}-\beta_{k}^{H S} g_{k}^{T} d_{k-1}+\beta_{k}^{H S} g_{k}^{T} d_{k-1}=-\left\|g_{k}\right\|^{2},
$$

which is independent of any line search and convexity of the objective function. In this case, Algorithm 2.2 reduces to the three-term HS method [24].

Remark 2.2. If exact line search is used, it is easy to see that Algorithm 2.1 and Algorithm 2.2 reduce to the standard HS method.

\section{Convergence properties}

In this section, we only analyze convergence properties of Algorithm 2.1. The corresponding results for Algorithm 2.2 can be obtained by using same argument as Algorithm 2.1. In the global convergence analysis of many iterative methods, the following assumption is often needed.

\section{Assumption A.}

(i) The level set $\Omega=\left\{x \in R^{n} \mid f(x) \leq f\left(x_{0}\right)\right\}$ is bounded.

(ii) In some neighborhood $N$ of $\Omega, f$ is continuously differentiable and its gradient is Lipschitz continuous, namely, there exists a constant $L>0$ such that

$$
\|g(x)-g(y)\| \leq L\|x-y\|, \quad \forall x, y \in N
$$

Clearly, Assumption A implies that there exists a constant $\gamma$ such that

$$
\|g(x)\| \leq \gamma, \quad \text { for all } \quad x \in N .
$$

In order to ensure global convergence of Algorithm 2.1, we need some line search to compute the stepsize $\alpha_{k}$. The Wolfe line search consists of finding $\alpha_{k}$ satisfying

$$
\left\{\begin{array}{l}
f\left(x_{k}+\alpha_{k} d_{k}\right) \leq f\left(x_{k}\right)+\delta \alpha_{k} g_{k}^{T} d_{k}, \\
d_{k}^{T} g\left(x_{k}+\alpha_{k} d_{k}\right) \geq \sigma d_{k}^{T} g_{k} .
\end{array}\right.
$$


The strong Wolfe line search corresponds to: that

$$
\left\{\begin{array}{l}
f\left(x_{k}+\alpha_{k} d_{k}\right) \leq f\left(x_{k}\right)+\delta \alpha_{k} g_{k}^{T} d_{k}, \\
\left|d_{k}^{T} g\left(x_{k}+\alpha_{k} d_{k}\right)\right| \leq\left|\sigma d_{k}^{T} g_{k}\right|,
\end{array}\right.
$$

where $0<\delta<\sigma<1$ are constants.

The following lemma, called the Zoutendijk condition, is often used to prove global convergence of conjugate gradient methods. It was originally given by Zoutendijk [25] and Wolfe [20].

Lemma 3.1. Let Assumption A hold, $\left\{x_{k}\right\}$ be generated by (1.2) and $d_{k}$ satisfy $g_{k}^{T} d_{k}<0$. If $\alpha_{k}$ satisfies the Wolfe condition (3.3) or the strong Wolfe condition (3.4), then we have

$$
\sum_{k=0}^{\infty} \frac{\left(g_{k}^{T} d_{k}\right)^{2}}{\left\|d_{k}\right\|^{2}}<+\infty
$$

In the global convergence analysis for many methods, the sufficient descent condition plays an important role. The following result shows that Algorithm 2.1 produces sufficient descent directions.

Lemma 3.2. Let $\left\{x_{k}\right\}$ and $\left\{d_{k}\right\}$ be generated by Algorithm 2.1, and let $\alpha_{k}$ be obtained by the Wolfe line search (3.4). If $\rho \in[0,1)$, then we have

$$
\frac{g_{k}^{T} d_{k}}{\left\|g_{k}\right\|^{2}} \leq-(1-\rho)
$$

Moreover, if $\rho=1$, then $g_{k}^{T} d_{k}<0$.

Proof. We have from (2.7) and the definition of $\beta_{k}^{H S}(1.4)$ that

$$
g_{k}^{T} d_{k}=-\left\|g_{k}\right\|^{2}+\rho \frac{g_{k}^{T} d_{k-1}}{d_{k-1}^{T} y_{k-1}}\left\|g_{k}\right\|^{2},
$$

which implies that

$$
\begin{aligned}
\frac{g_{k}^{T} d_{k}}{\left\|g_{k}\right\|^{2}} & =-1+\rho \frac{g_{k}^{T} d_{k-1}}{d_{k-1}^{T} y_{k-1}}=-(1-\rho)-\rho+\rho \frac{g_{k}^{T} d_{k-1}}{d_{k-1}^{T} y_{k-1}} \\
& =-(1-\rho)+\rho \frac{g_{k-1}^{T} d_{k-1}}{d_{k-1}^{T} y_{k-1}} .
\end{aligned}
$$


Since

$$
g_{0}^{T} d_{0}=-\left\|g_{0}\right\|^{2}<0
$$

and the Wolfe line search implies

$$
d_{0}^{T} y_{0} \geq(1-\sigma)\left\|g_{0}\right\|^{2}>0,
$$

from (3.8), we have (3.6) by induction. The proof is then finished.

By the use of the first equality in (3.8) and the second inequality in the strong Wolfe line search (3.4), we have the following result.

Lemma 3.3. Let $\left\{x_{k}\right\}$ and $\left\{d_{k}\right\}$ be generated by Algorithm 2.1, and let $\alpha_{k}$ be obtained by the strong Wolfe line search (3.4) with $\sigma<\frac{1}{2}$. If $\rho=1$, then we have

$$
\frac{g_{k}^{T} d_{k}}{\left\|g_{k}\right\|^{2}} \leq-\frac{1-2 \sigma}{1-\sigma}
$$

The following theorem establishes global convergence of Algorithm 2.1 for strongly convex functions.

Theorem 3.4. Suppose Assumption $A$ holds and $f$ is strongly convex on $N$, that is, there exists a constant $\mu>0$ such that

$$
(g(x)-g(y))^{T}(x-y) \geq \mu\|x-y\|^{2}, \quad \forall x, y \in N .
$$

If $\rho \in[0,1)$, then the sequence $\left\{x_{k}\right\}$ generated by Algorithm 2.1 with the Wolfe line search (3.3) satisfies $\lim _{k \rightarrow \infty}\left\|g_{k}\right\|=0$.

Proof. It follows from (3.10) and (3.1) that

$$
L d_{k-1}^{T} s_{k-1} \geq d_{k-1}^{T} y_{k-1} \geq \mu d_{k-1}^{T} s_{k-1} .
$$

Now we begin to estimate $\theta_{k}$ and $\beta_{k}$ in (2.7). It follows from (1.4), (3.1) and (3.11) that

$$
\left|\beta_{k}^{H S}\right|=\left|\frac{g_{k}^{T} y_{k-1}}{d_{k-1}^{T} y_{k-1}}\right| \leq \frac{L\left\|g_{k}\right\|\left\|s_{k-1}\right\|}{\mu d_{k-1}^{T} s_{k-1}}=\frac{L\left\|g_{k}\right\|}{\mu\left\|d_{k-1}\right\|} .
$$


It follows from (2.8), (3.12) and the second inequality in (3.3) that

$$
\begin{aligned}
\left|\theta_{k}\right| & =\left|1-\rho+\beta_{k}^{H S} \frac{g_{k}^{T} d_{k-1}}{\left\|g_{k}\right\|^{2}}-\rho \frac{g_{k-1}^{T} d_{k-1}}{d_{k-1}^{T} y_{k-1}}\right| \\
& \leq 1-\rho+\left|\beta_{k}^{H S}\right| \frac{\left\|g_{k}\right\|\left\|d_{k-1}\right\|}{\left\|g_{k}\right\|^{2}}+\frac{\rho\left|g_{k-1}^{T} d_{k-1}\right|}{(1-\sigma)\left|g_{k-1}^{T} d_{k-1}\right|} \\
& \leq 1-\rho+\frac{L}{\mu}+\frac{\rho}{1-\sigma} .
\end{aligned}
$$

The above inequality together with (3.12) implies that

$$
\begin{aligned}
\left\|d_{k}\right\| & \leq\left|\theta_{k}\right|\left\|g_{k}\right\|+\left|\beta_{k}^{H S}\right|\left\|d_{k-1}\right\| \\
& \leq\left(1-\rho+2 \frac{L}{\mu}+\frac{\rho}{1-\sigma}\right)\left\|g_{k}\right\| \triangleq M\left\|g_{k}\right\| .
\end{aligned}
$$

We have from (3.6) and (3.5) that

$$
\sum_{k=0}^{\infty} \frac{\left\|g_{k}\right\|^{4}}{\left\|d_{k}\right\|^{2}}<\infty .
$$

It follows from the above inequality and (3.13) that

$$
\sum_{k=0}^{\infty}\left\|g_{k}\right\|^{2}<\infty
$$

which means $\lim _{k \rightarrow \infty}\left\|g_{k}\right\|=0$. The proof is then completed.

By Lemma 3.3 and same argument in the above theorem, we have the following corollary.

Corollary 3.5. Suppose Assumption A holds and $f$ is strongly convex on $N$. If $\rho=1$ and $\alpha_{k}$ is determined by the strong Wolfe line search (3.4) with $\sigma<\frac{1}{2}$, then the sequence $\left\{x_{k}\right\}$ generated by Algorithm 2.1 satisfies $\lim _{k \rightarrow \infty}\left\|g_{k}\right\|=0$.

In order to ensure global convergence of Algorithm 2.1 for nonconvex functions, we adopt the idea of the MBFGS method proposed by Li and Fukushima [14] and modify Algorithm 2.1, replacing $y_{k-1}$ in (2.7) by

$$
z_{k-1}=y_{k-1}+\varepsilon_{1} s_{k-1},
$$

where $\varepsilon_{1}$ is a small positive constant. For convenience, we present this modified version as the following algorithm. 
Algorithm 3.1 (modified two-term HS Method):

$$
d_{k}= \begin{cases}-g_{k}, & \text { if } k=0 \\ -\theta_{k}^{M H S} g_{k}+\beta_{k}^{M H S} d_{k-1}, & \text { if } k>0\end{cases}
$$

where

$$
\beta_{k}^{M H S}=\frac{g_{k}^{T} z_{k-1}}{d_{k-1}^{T} z_{k-1}}, \quad \theta_{k}^{M H S}=1+\beta_{k}^{M H S} \frac{g_{k}^{T} d_{k-1}}{\left\|g_{k}\right\|^{2}}-\rho \frac{g_{k}^{T} d_{k-1}}{d_{k-1}^{T} z_{k-1}} .
$$

An important property of $z_{k-1}$ is that, when the Wolfe line search is used, it satisfies

$$
\left(L+\varepsilon_{1}\right) d_{k-1}^{T} S_{k-1} \geq d_{k-1}^{T} z_{k-1} \geq \varepsilon_{1} d_{k-1}^{T} s_{k-1} .
$$

This inequality is the same as (3.11) and plays the same role in the proof of global convergence of Algorithm 3.1 for nonconvex functions. By the use of (3.17) and the same arguments as in Theorem 3.4, we have the following strongly global convergence result for Algorithm 3.1 for nonconvex objective functions.

Theorem 3.6. Suppose Assumption A holds, then the sequence $\left\{x_{k}\right\}$ generated by Algorithm 3.1 with the Wolfe line search (3.3) satisfies $\lim _{k \rightarrow \infty}\left\|g_{k}\right\|=0$.

Another technique to guarantee global convergence of conjugate gradient methods for general nonlinear functions is to restrict $\beta_{k}$ nonnegative as in the $\mathrm{PRP}+$ and HS + methods [10]. In fact, if we replace $\beta_{k}^{H S}$ in Algorithm 2.1 by $\beta_{k}^{H S+}=\max \left\{0, \beta_{k}^{H S}\right\}$, we have the following algorithm which we call the twoterm HS+ Method.

Algorithm 3.2 (two-term HS+ Method):

$$
d_{k}= \begin{cases}-g_{k}, & \text { if } k=0, \\ -\theta_{k}^{H S+} g_{k}+\beta_{k}^{H S+} d_{k-1}, & \text { if } k>0,\end{cases}
$$

where

$$
\theta_{k}^{H S+}=1+\beta_{k}^{H S+} \frac{g_{k}^{T} d_{k-1}}{\left\|g_{k}\right\|^{2}}-\rho \frac{g_{k}^{T} d_{k-1}}{d_{k-1}^{T} y_{k-1}} .
$$

Using the same argument as Theorem 4.3 in [10], we can get the following global convergence result. Here we omit its proof. 
Theorem 3.7. Suppose Assumption A holds, then the sequence $\left\{x_{k}\right\}$ generated by Algorithm 3.2 with the strong Wolfe line search (3.4) satisfies

$$
\liminf _{k \rightarrow \infty}\left\|g_{k}\right\|=0 \text {. }
$$

\section{Applications}

In this section, we extend the results on new versions of the HS method in Sections 2 and 3 to some well-known conjugate gradient methods. For instance, if we replace the term $d_{k-1}^{T} y_{k-1}$ in step 1 of Algorithm 2.1 and Algorithm 2.2 by $\left\|g_{k-1}\right\|^{2}$ or $-d_{k-1}^{T} g_{k-1}$, we get new versions of the PRP and LS methods, respectively.

Algorithm 4.1 (two-term PRP Method):

$$
d_{k}= \begin{cases}-g_{k}, & \text { if } k=0, \\ -\theta_{k}^{P R P 1} g_{k}+\beta_{k}^{P R P} d_{k-1}, & \text { if } k>0,\end{cases}
$$

where

$$
\theta_{k}^{P R P 1}=1+\beta_{k}^{P R P} \frac{g_{k}^{T} d_{k-1}}{\left\|g_{k}\right\|^{2}}-\rho \frac{g_{k}^{T} d_{k-1}}{\left\|g_{k-1}\right\|^{2}} .
$$

Algorithm 4.2 (three-term PRP Method):

$$
d_{k}= \begin{cases}-g_{k}, & \text { if } k=0, \\ -g_{k}+\beta_{k}^{P R P} d_{k-1}+\theta_{k}^{P R P 2} y_{k-1}, & \text { if } k>0,\end{cases}
$$

where

$$
\theta_{k}^{P R P 2}=\rho \frac{\left\|g_{k}\right\|^{2}}{g_{k}^{T} y_{k-1}} \frac{g_{k}^{T} d_{k-1}}{\left\|g_{k-1}\right\|^{2}}-\frac{g_{k}^{T} d_{k-1}}{\left\|g_{k-1}\right\|^{2}} .
$$

Algorithm 4.3 (two-term LS Method):

$$
d_{k}= \begin{cases}-g_{k}, & \text { if } k=0, \\ -\theta_{k}^{L S 1} g_{k}+\beta_{k}^{L S} d_{k-1}, & \text { if } k>0,\end{cases}
$$

where

$$
\beta_{k}^{L S}=-\frac{g_{k}^{T} y_{k-1}}{d_{k-1}^{T} g_{k-1}}, \quad \theta_{k}^{L S 1}=1+\beta_{k}^{L S} \frac{g_{k}^{T} d_{k-1}}{\left\|g_{k}\right\|^{2}}+\rho \frac{g_{k}^{T} d_{k-1}}{d_{k-1}^{T} g_{k-1}} .
$$


Algorithm 4.4 (three-term LS Method):

$$
d_{k}= \begin{cases}-g_{k}, & \text { if } k=0, \\ -g_{k}+\beta_{k}^{L S} d_{k-1}+\theta_{k}^{L S 2} y_{k-1}, & \text { if } k>0\end{cases}
$$

where

$$
\theta_{k}^{L S 2}=-\rho \frac{\left\|g_{k}\right\|^{2}}{g_{k}^{T} y_{k-1}} \frac{g_{k}^{T} d_{k-1}}{d_{k-1}^{T} g_{k-1}}+\frac{g_{k}^{T} d_{k-1}}{d_{k-1}^{T} g_{k-1}} .
$$

Algorithm 4.5 (two-term FR Method):

$$
d_{k}= \begin{cases}-g_{k}, & \text { if } k=0, \\ -\theta_{k}^{F R} g_{k}+\beta_{k}^{F R} d_{k-1}, & \text { if } k>0,\end{cases}
$$

where

$$
\theta_{k}^{F R}=1+\beta_{k}^{F R} \frac{g_{k}^{T} d_{k-1}}{\left\|g_{k}\right\|^{2}}-\rho \frac{g_{k}^{T} d_{k-1}}{\left\|g_{k-1}\right\|^{2}}
$$

Remark 4.1. When $\rho=0$ in the above algorithms, the search direction satisfies the sufficient descent condition $d_{k}^{T} g_{k}=-\left\|g_{k}\right\|^{2}$, which is also independent of any line search and convexity of the objective function. Moreover in this case, Algorithms 4.2 and 4.3 are identical and reduce to the modified PRP method [23], and Algorithm 4.5 becomes the modified FR method [22]. It is clear that these methods reduce to conjugate gradient methods respectively if exact line search is used.

Remark 4.2. Global convergence properties of these algorithms are similar to those of Algorithm 2.1 or Algorithm 2.2. Here we only analyze Algorithm 4.1 and Algorithm 4.5.

The next result shows that the direction generated by Algorithm 4.1 or Algorithm 4.5 satisfies the sufficient descent condition if the strong Wolfe line search (3.4) is used.

Lemma 4.1. Let $\left\{x_{k}\right\}$ and $\left\{d_{k}\right\}$ be generated by Algorithm 4.1 or Algorithm 4.5 with the strong Wolfe line search (3.4). If $\rho<\frac{1}{2 \sigma}$, then for all $k$, we have that

$$
-\frac{1-(\rho \sigma)^{k}}{1-\rho \sigma} \leq \frac{g_{k}^{T} d_{k}}{\left\|g_{k}\right\|^{2}} \leq-\frac{1-2 \rho \sigma+(\rho \sigma)^{k}}{1-\rho \sigma} \leq-\frac{1-2 \rho \sigma}{1-\rho \sigma} .
$$




\begin{tabular}{|c|c|c|c|c|c|c|c|}
\hline problem & $\rho$ & iter & fn & gn & $\|g(x)\|_{\infty}$ & $f(x)$ & time \\
\hline \multirow{6}{*}{$\begin{array}{l}\text { DIXMAANI } \\
(6000)\end{array}$} & 0.0 & 3459 & 6919 & 3460 & $0.9641 \mathrm{D}-06$ & $0.1000 \mathrm{D}+01$ & 13.58 \\
\hline & 0.2 & 3376 & 6753 & 3377 & $0.9525 \mathrm{D}-06$ & $0.1000 \mathrm{D}+01$ & 12.95 \\
\hline & 0.4 & 3241 & 6483 & 3242 & $0.9934 \mathrm{D}-06$ & $0.1000 \mathrm{D}+01$ & 12.44 \\
\hline & 0.6 & 3074 & 6149 & 3075 & $0.9505 \mathrm{D}-06$ & $0.1000 \mathrm{D}+01$ & 11.79 \\
\hline & 0.8 & 2927 & 5855 & 2928 & $0.9941 \mathrm{D}-06$ & $0.1000 \mathrm{D}+01$ & 11.22 \\
\hline & 1.0 & 2870 & 5741 & 2871 & $0.9970 \mathrm{D}-06$ & $0.1000 \mathrm{D}+01$ & 11.01 \\
\hline \multirow{6}{*}{$\begin{array}{l}\text { TRIDIA } \\
(10000)\end{array}$} & 0.0 & 1115 & 2231 & 1116 & $0.9932 \mathrm{D}-06$ & $0.4211 \mathrm{D}-14$ & 2.92 \\
\hline & 0.2 & 1115 & 2231 & 1116 & $0.9907 \mathrm{D}-06$ & $0.4216 \mathrm{D}-14$ & 3.71 \\
\hline & 0.4 & 1115 & 2231 & 1116 & $0.9982 \mathrm{D}-06$ & $0.4181 \mathrm{D}-14$ & 2.91 \\
\hline & 0.6 & 1116 & 2233 & 1117 & $0.9628 \mathrm{D}-06$ & $0.3884 \mathrm{D}-14$ & 2.93 \\
\hline & 0.8 & 1115 & 2231 & 1116 & $0.9982 \mathrm{D}-06$ & $0.4185 \mathrm{D}-14$ & 2.93 \\
\hline & 1.0 & 1115 & 2231 & 1116 & $0.9681 \mathrm{D}-06$ & $0.4176 \mathrm{D}-14$ & 2.92 \\
\hline \multirow{6}{*}{$\begin{array}{l}\text { CURLY30 } \\
(1000)\end{array}$} & 0.0 & 10769 & 17008 & 17441 & $0.8923 \mathrm{D}-06$ & $-0.1003 D+06$ & 10.01 \\
\hline & 0.2 & 9993 & 16126 & 15621 & $0.8650 \mathrm{D}-06$ & $-0.1003 D+06$ & 8.76 \\
\hline & 0.4 & 10706 & 16996 & 17134 & $0.8343 \mathrm{D}-06$ & $-0.1003 D+06$ & 9.51 \\
\hline & 0.6 & 10065 & 16310 & 15707 & $0.9882 \mathrm{D}-06$ & $-0.1003 D+06$ & 8.84 \\
\hline & 0.8 & 9854 & 15998 & 15246 & $0.9443 \mathrm{D}-06$ & $-0.1003 D+06$ & 8.61 \\
\hline & 1.0 & 9808 & 15994 & 15012 & $0.9442 \mathrm{D}-06$ & $-0.1003 D+06$ & 8.52 \\
\hline \multirow{6}{*}{$\begin{array}{l}\text { CURLY10 } \\
(1000)\end{array}$} & 0.0 & 10853 & 16209 & 17260 & $0.9280 \mathrm{D}-06$ & $-0.1003 D+06$ & 5.05 \\
\hline & 0.2 & 10867 & 16213 & 17244 & $0.9981 \mathrm{D}-06$ & $-0.1003 D+06$ & 4.86 \\
\hline & 0.4 & 10660 & 15986 & 16848 & $0.8842 \mathrm{D}-06$ & $-0.1003 D+06$ & 4.80 \\
\hline & 0.6 & 10781 & 16127 & 17078 & $0.9710 \mathrm{D}-06$ & $-0.1003 D+06$ & 4.85 \\
\hline & 0.8 & 10743 & 16062 & 16999 & $0.9429 \mathrm{D}-06$ & $-0.1003 D+06$ & 4.84 \\
\hline & 1.0 & 10732 & 15957 & 17087 & $0.9225 \mathrm{D}-06$ & $-0.1003 D+06$ & 4.84 \\
\hline \multirow{6}{*}{$\begin{array}{l}\text { EIGENALS } \\
\text { ( } 930)\end{array}$} & 0.0 & 2755 & 5516 & 2767 & 0.8979D-06 & 0.4309D-10 & 26.27 \\
\hline & 0.2 & 2921 & 5848 & 2934 & $0.5473 \mathrm{D}-06$ & $0.3583 \mathrm{D}-10$ & 21.69 \\
\hline & 0.4 & 2603 & 5212 & 2615 & $0.7806 \mathrm{D}-06$ & $0.6556 \mathrm{D}-10$ & 19.68 \\
\hline & 0.6 & 2838 & 5682 & 2848 & $0.8246 \mathrm{D}-06$ & $0.5192 \mathrm{D}-10$ & 21.07 \\
\hline & 0.8 & 2639 & 5284 & 2653 & $0.9181 \mathrm{D}-06$ & $0.2030 \mathrm{D}-10$ & 19.98 \\
\hline & 1.0 & 2704 & 5414 & 2715 & $0.5283 \mathrm{D}-06$ & $0.3591 \mathrm{D}-10$ & 20.04 \\
\hline \multirow{6}{*}{$\begin{array}{l}\text { FMINSURF } \\
(10000)\end{array}$} & 0.0 & 607 & 1218 & 611 & $0.9973 \mathrm{D}-06$ & $0.1000 \mathrm{D}+01$ & 5.54 \\
\hline & 0.2 & 629 & 1259 & 630 & 0.8559D-06 & $0.1000 \mathrm{D}+01$ & 5.29 \\
\hline & 0.4 & 609 & 1219 & 610 & $0.8983 \mathrm{D}-06$ & $0.1000 \mathrm{D}+01$ & 5.12 \\
\hline & 0.6 & 601 & 1203 & 602 & $0.9117 \mathrm{D}-06$ & $0.1000 \mathrm{D}+01$ & 5.07 \\
\hline & 0.8 & 600 & 1201 & 601 & $0.8677 \mathrm{D}-06$ & $0.1000 \mathrm{D}+01$ & 5.07 \\
\hline & 1.0 & 590 & 1182 & 592 & $0.9383 \mathrm{D}-06$ & $0.1000 \mathrm{D}+01$ & 4.97 \\
\hline \multirow{6}{*}{$\begin{array}{l}\text { BDQRTIC } \\
(10000)\end{array}$} & 0.0 & 10006 & 14943 & 21460 & $0.8604 \mathrm{D}-06$ & $0.4003 \mathrm{D}+05$ & 116.72 \\
\hline & 0.2 & 2031 & 4188 & 3071 & $0.9234 \mathrm{D}-06$ & $0.4003 \mathrm{D}+05$ & 20.47 \\
\hline & 0.4 & 2636 & 5530 & 4672 & $0.9835 \mathrm{D}-06$ & $0.4003 \mathrm{D}+05$ & 29.34 \\
\hline & 0.6 & 1359 & 2986 & 2498 & $0.8684 \mathrm{D}-06$ & $0.4003 \mathrm{D}+05$ & 15.85 \\
\hline & 0.8 & 1247 & 2733 & 2394 & $0.8808 \mathrm{D}-06$ & $0.4003 \mathrm{D}+05$ & 14.18 \\
\hline & 1.0 & 1879 & 4036 & 4982 & $0.9410 \mathrm{D}-06$ & $0.4003 \mathrm{D}+05$ & 27.80 \\
\hline \multirow{6}{*}{$\begin{array}{l}\text { FMINSRF2 } \\
(10000)\end{array}$} & 0.0 & 423 & 848 & 425 & $0.9950 \mathrm{D}-06$ & $0.1000 \mathrm{D}+01$ & 3.73 \\
\hline & 0.2 & 435 & 871 & 436 & $0.9353 \mathrm{D}-06$ & $0.1000 \mathrm{D}+01$ & 3.86 \\
\hline & 0.4 & 420 & 841 & 421 & $0.9953 \mathrm{D}-06$ & $0.1000 \mathrm{D}+01$ & 3.45 \\
\hline & 0.6 & 420 & 841 & 421 & $0.9892 \mathrm{D}-06$ & $0.1000 \mathrm{D}+01$ & 3.46 \\
\hline & 0.8 & 426 & 853 & 427 & $0.8962 \mathrm{D}-06$ & $0.1000 \mathrm{D}+01$ & 3.51 \\
\hline & 1.0 & 420 & 841 & 421 & $0.8994 \mathrm{D}-06$ & $0.1000 \mathrm{D}+01$ & 3.46 \\
\hline
\end{tabular}

Table 1 - Test results for Algorithm 2.1 with different $\rho$ values. 


\begin{tabular}{lcclcccc}
\hline \multicolumn{1}{c}{ problem } & $\rho$ & iter & \multicolumn{1}{c}{ fn } & gn & $\|g(x)\|_{\infty}$ & $f(x)$ & time \\
\hline FMINSRF2 & 0.0 & 495 & 995 & 500 & $0.9745 \mathrm{D}-06$ & $0.1000 \mathrm{D}+01$ & 7.53 \\
$(15625)$ & 0.2 & 497 & 997 & 500 & $0.9647 \mathrm{D}-06$ & $0.1000 \mathrm{D}+01$ & 6.76 \\
& 0.4 & 507 & 1015 & 508 & $0.9508 \mathrm{D}-06$ & $0.1000 \mathrm{D}+01$ & 7.07 \\
& 0.6 & 491 & 984 & 493 & $0.9896 \mathrm{D}-06$ & $0.1000 \mathrm{D}+01$ & 6.70 \\
& 0.8 & 515 & 1031 & 516 & $0.9639 \mathrm{D}-06$ & $0.1000 \mathrm{D}+01$ & 6.86 \\
& 1.0 & 492 & 985 & 493 & $0.8987 \mathrm{D}-06$ & $0.1000 \mathrm{D}+01$ & 6.54 \\
TESTQUAD & 0.0 & 2213 & 4427 & 2214 & $0.8599 \mathrm{D}-06$ & $0.2117 \mathrm{D}-12$ & 5.50 \\
$(10000)$ & 0.2 & 2227 & 4455 & 2228 & $0.9356 \mathrm{D}-06$ & $0.2108 \mathrm{D}-12$ & 5.07 \\
& 0.4 & 2169 & 4339 & 2170 & $0.9682 \mathrm{D}-06$ & $0.7841 \mathrm{D}-12$ & 4.83 \\
& 0.6 & 2232 & 4465 & 2233 & $0.8829 \mathrm{D}-06$ & $0.2106 \mathrm{D}-12$ & 4.91 \\
& 0.8 & 2175 & 4351 & 2176 & $0.9072 \mathrm{D}-06$ & $0.7838 \mathrm{D}-12$ & 4.77 \\
& 1.0 & 2234 & 4469 & 2235 & $0.8896 \mathrm{D}-06$ & $0.2107 \mathrm{D}-12$ & 4.90 \\
\hline
\end{tabular}

Table $1-$ (continuation).

Proof. We prove (4.5) by induction. Since $g_{0}^{T} d_{0}=-\left\|g_{0}\right\|^{2}$, in this case, the relation (4.5) holds with $k=0$. It follows from (4.1) or (4.3) that

$$
\frac{g_{k}^{T} d_{k}}{\left\|g_{k}\right\|^{2}}=-1+\rho \frac{g_{k}^{T} d_{k-1}}{\left\|g_{k-1}\right\|^{2}} .
$$

The above equality with the second inequality in (3.4) implies that

$$
-1+\rho \sigma \frac{g_{k-1}^{T} d_{k-1}}{\left\|g_{k-1}\right\|^{2}} \leq \frac{g_{k}^{T} d_{k}}{\left\|g_{k}\right\|^{2}} \leq-1-\rho \sigma \frac{g_{k-1}^{T} d_{k-1}}{\left\|g_{k-1}\right\|^{2}} .
$$

Repeating the same process, we have that $-\left(1+\rho \sigma+(\rho \sigma)^{2}+\cdots+(\rho \sigma)^{k-1}\right) \leq \frac{g_{k}^{T} d_{k}}{\left\|g_{k}\right\|^{2}} \leq-1+\rho \sigma+(\rho \sigma)^{2}+\cdots+(\rho \sigma)^{k-1}$,

which shows that (4.5) holds. The proof is then completed.

Remark 4.3. If we replace $\beta_{k}^{P R P}$ in Algorithm 4.1 by $\beta_{k}^{P R P+}=\max \left\{0, \beta_{k}^{P R P}\right\}$, then this restricted algorithm converges globally for nonconvex functions by Lemma 4.1 and the argument of Theorem 4.3 in [10].

The next result is based on the work of [23]. Here we also omit its proof.

Theorem 4.2. Suppose Assumption A holds. Let $\left\{x_{k}\right\}$ and $\left\{d_{k}\right\}$ be generated by Algorithm 4.5 with the strong Wolfe line search (3.4). If $\rho<\frac{1}{2 \sigma}$, then we have $\liminf _{k \rightarrow \infty}\left\|g_{k}\right\|=0$. 


\begin{tabular}{|c|c|c|c|c|}
\hline & & cg-descent & Algorithm 2.1 & prp + \\
\hline problem & $\mathrm{n}$ & iter / fn / gn /time & iter / fn / gn / time & iter / fn / gn /time \\
\hline FLETCHCR & 5000 & $31998 / 66391 / 34677 / 102.18$ & $20046 / 40142 / 20108 / 59.04$ & $19987 / 40000 / 40000 / 83.00$ \\
\hline CURLY30 & 1000 & $9765 / 15713 / 15122 / 8.51$ & $9808 / 15994 / 15012 / 8.45$ & $-1 /-1 /-1 /-1.00$ \\
\hline CURLY20 & 1000 & $9757 / 15481 / 15084 / 6.75$ & $10644 / 16517 / 17037 / 7.22$ & $-1 /-1 /-1 /-1.00$ \\
\hline DIXMAANI & 6000 & $2660 / 5321 / 2661 / 10.65$ & $2870 / 5741 / 2871 / 11.10$ & $2357 / 4720 / 4720 / 14.10$ \\
\hline EIGENBLS & 420 & 4970/9947/ 4978/ 9.39 & $4615 / 9236 / 4623 / 8.54$ & $4832 / 9714 / 9714 / 14.91$ \\
\hline TRIDIA & 10000 & $1115 / 2231 / 1116 / 3.02$ & $1115 / 2231 / 1116 / 3.22$ & $1114 / 2231 / 2231 / 3.91$ \\
\hline NONDQUAR & 5000 & $5012 / 10050 / 5099 / 7.43$ & $2994 / 6004 / 3104 / 4.25$ & $5006 / 10058 / 10058 / 9.52$ \\
\hline CURLY10 & 1000 & $9431 / 14475 / 14406 / 4.48$ & $10732 / 15957 / 17087 / 4.81$ & $-1 /-1 /-1 /-1.00$ \\
\hline EIGENCLS & 462 & $1776 / 3575 / 1802 / 3.94$ & $1729 / 3467 / 1741 / 3.98$ & $1650 / 3312 / 3312 / 6.00$ \\
\hline SPARSINE & 1000 & $4515 / 9031 / 4516 / 4.17$ & $6091 / 12183 / 6092 / 6.06$ & $4394 / 8793 / 8793 / 6.44$ \\
\hline EIGENALS & 420 & $1329 / 2665 / 1344 / 2.52$ & $1336 / 2679 / 1353 / 2.73$ & $1482 / 2998 / 2998 / 4.63$ \\
\hline FLETCHCR & 1000 & $6828 / 14236 / 7479 / 3.99$ & $4939 / 9987 / 5062 / 2.41$ & $4471 / 8986 / 8986 / 3.37$ \\
\hline GENHUMPS & 1000 & $3344 / 6857 / 3555 / 6.35$ & $2486 / 5046 / 2580 / 4.33$ & $2719 / 5807 / 5807 / 7.48$ \\
\hline FMINSURF & 5625 & $491 / 983 / 492 / 2.14$ & $475 / 954 / 479 / 2.31$ & $471 / 949 / 949 / 3.25$ \\
\hline TRIDIA & 5000 & $782 / 1565 / 783 / 0.79$ & $782 / 1565 / 783 / 0.86$ & $781 / 1565 / 1565 / 1.29$ \\
\hline DIXMAANE & 6000 & $302 / 605 / 303 / 1.23$ & $306 / 613 / 307 / 1.32$ & $307 / 620 / 620 / 2.09$ \\
\hline DIXMAANJ & 6000 & $295 / 591 / 296 / 1.25$ & $311 / 623 / 312 / 1.32$ & $275 / 557 / 557 / 1.99$ \\
\hline BDQRTIC & 5000 & $3763 / 6992 / 8726 / 22.78$ & $1706 / 3547 / 2102 / 6.90$ & $-1 /-1 /-1 /-1.00$ \\
\hline DIXMAANK & 6000 & $263 / 527 / 264 / 1.05$ & $314 / 629 / 315 / 1.23$ & $289 / 587 / 587 / 1.77$ \\
\hline NONCVXU2 & 1000 & $1928 / 3731 / 2055 / 2.14$ & $1942 / 3785 / 2043 / 1.80$ & $1956 / 3919 / 3919 / 2.81$ \\
\hline DIXMAANL & 6000 & $244 / 489 / 245 / 1.02$ & $264 / 529 / 265 / 1.09$ & $346 / 702 / 702 / 2.65$ \\
\hline SENSORS & 100 & $25 / 57 / 44 / 0.78$ & $31 / 67 / 49 / 0.95$ & $27 / 66 / 66 / 1.17$ \\
\hline DIXMAANF & 6000 & $229 / 459 / 230 / 0.98$ & $223 / 447 / 224 / 1.02$ & $215 / 437 / 437 / 1.62$ \\
\hline DIXMAANG & 6000 & $226 / 453 / 227 / 0.96$ & $228 / 457 / 229 / 0.96$ & $206 / 420 / 420 / 1.81$ \\
\hline DIXMAANH & 6000 & $223 / 447 / 224 / 0.95$ & $218 / 437 / 219 / 0.93$ & $408 / 825 / 825 / 2.97$ \\
\hline FLETCBV2 & 1000 & $1052 / 2105 / 1055 / 0.94$ & $1222 / 2445 / 1225 / 1.06$ & $942 / 1886 / 1886 / 1.50$ \\
\hline SCHMVETT & 10000 & $41 / 68 / 60 / 1.37$ & $50 / 87 / 73 / 1.64$ & $44 / 105 / 105 / 2.45$ \\
\hline GENHUMPS & 500 & $1940 / 4132 / 2258 / 1.90$ & $2032 / 4179 / 2171 / 1.82$ & $1896 / 4147 / 4147 / 2.60$ \\
\hline CRAGGLVY & 5000 & $111 / 204 / 143 / 0.98$ & $110 / 204 / 150 / 1.19$ & $-1 /-1 /-1 /-1.00$ \\
\hline MOREBV & 10000 & $95 / 191 / 97 / 0.67$ & $106 / 213 / 108 / 0.77$ & $100 / 201 / 201 / 1.33$ \\
\hline WOODS & 10000 & $187 / 426 / 257 / 1.18$ & $224 / 491 / 283 / 1.31$ & $232 / 487 / 487 / 2.15$ \\
\hline NONDQUAR & 1000 & $2958 / 5924 / 3147 / 0.87$ & $3059 / 6128 / 3291 / 0.87$ & $4015 / 8128 / 8128 / 1.51$ \\
\hline SPARSQUR & 10000 & $22 / 45 / 23 / 0.37$ & $34 / 69 / 35 / 0.53$ & $41 / 131 / 131 / 1.42$ \\
\hline POWER & 5000 & $258 / 517 / 259 / 0.31$ & $262 / 525 / 263 / 0.29$ & $252 / 514 / 514 / 0.40$ \\
\hline MANCINO & 100 & $11 / 23 / 12 / 0.66$ & $12 / 25 / 13 / 0.71$ & $11 / 27 / 27 / 1.09$ \\
\hline CRAGGLVY & 2000 & $106 / 191 / 132 / 0.35$ & $108 / 200 / 140 / 0.37$ & $-1 /-1 /-1 /-1.00$ \\
\hline CURLY30 & 200 & $1920 / 3515 / 2819 / 0.33$ & $1810 / 3330 / 2594 / 0.30$ & $-1 /-1 /-1 /-1.00$ \\
\hline LIARWHD & 10000 & $25 / 60 / 41 / 0.42$ & $19 / 40 / 24 / 0.34$ & $15 / 46 / 46 / 0.41$ \\
\hline BDQRTIC & 1000 & $628 / 1296 / 1025 / 0.56$ & $416 / 878 / 572 / 0.35$ & $-1 /-1 /-1 /-1.00$ \\
\hline GENROSE & 500 & $1259 / 2559 / 1309 / 0.33$ & $1163 / 2350 / 1207 / 0.26$ & $1123 / 2278 / 2278 / 0.36$ \\
\hline VARDIM & 10000 & $50 / 109 / 62 / 0.31$ & $52 / 138 / 91 / 0.34$ & $-1 /-1 /-1 /-1.00$ \\
\hline CURLY20 & 200 & $1998 / 3546 / 2951 / 0.28$ & $1895 / 3386 / 2769 / 0.25$ & $-1 /-1 /-1 /-1.00$ \\
\hline FREUROTH & 5000 & $65 / 126 / 96 / 0.48$ & $69 / 134 / 108 / 0.52$ & $-1 /-1 /-1 /-1.00$ \\
\hline ENGVAL1 & 10000 & $25 / 43 / 35 / 0.36$ & $25 / 47 / 35 / 0.37$ & $-1 /-1 /-1 /-1.00$ \\
\hline POWELLSG & 10000 & $64 / 130 / 77 / 0.29$ & $187 / 378 / 216 / 0.62$ & $165 / 362 / 362 / 0.73$ \\
\hline DIXON3DQ & 1000 & $1000 / 2001 / 1002 / 0.26$ & $1000 / 2001 / 1002 / 0.24$ & $1000 / 2005 / 2005 / 0.33$ \\
\hline BRYBND & 5000 & $36 / 74 / 39 / 0.31$ & $32 / 66 / 35 / 0.28$ & $26 / 66 / 66 / 0.38$ \\
\hline HILBERTA & 200 & $40 / 81 / 50 / 0.45$ & $16 / 33 / 21 / 0.29$ & $15 / 38 / 38 / 0.35$ \\
\hline TQUARTIC & 10000 & $25 / 77 / 61 / 0.59$ & $17 / 86 / 76 / 0.63$ & $13 / 38 / 38 / 0.50$ \\
\hline CURLY10 & 200 & $2127 / 3649 / 3100 / 0.20$ & $2115 / 3640 / 3079 / 0.20$ & $-1 /-1 /-1 /-1.00$ \\
\hline
\end{tabular}

Table 2 - Test results of the proposed methods. 


\begin{tabular}{|c|c|c|c|c|}
\hline & & cg-descent & Algorithm 2.1 & prp+ \\
\hline problem & $\mathrm{n}$ & iter / fn / gn /time & iter / fn / gn / time & iter / fn / gn /time \\
\hline FLETCBV2 & 500 & $478 / 957 / 480 / 0.24$ & $481 / 963 / 483 / 0.23$ & $480 / 962 / 962 / 0.32$ \\
\hline EDENSCH & 5000 & $34 / 61 / 43 / 0.29$ & $33 / 60 / 41 / 0.25$ & $-1 /-1 /-1 /-1.00$ \\
\hline MOREBV & 1000 & $425 / 851 / 426 / 0.22$ & $425 / 851 / 426 / 0.22$ & $425 / 851 / 851 / 0.31$ \\
\hline VAREIGVL & 5000 & $147 / 295 / 148 / 1.12$ & $161 / 323 / 162 / 1.20$ & $147 / 304 / 304 / 1.71$ \\
\hline PENALTY1 & 10000 & $65 / 159 / 104 / 0.48$ & $51 / 115 / 70 / 0.38$ & $14 / 81 / 81 / 0.35$ \\
\hline QUARTC & 10000 & $35 / 71 / 36 / 0.18$ & $51 / 103 / 52 / 0.23$ & $16 / 69 / 69 / 0.20$ \\
\hline FMINSURF & 1024 & $236 / 474 / 238 / 0.19$ & $238 / 477 / 239 / 0.19$ & $226 / 455 / 455 / 0.27$ \\
\hline VARDIM & 5000 & $43 / 87 / 44 / 0.12$ & $44 / 89 / 46 / 0.11$ & $-1 /-1 /-1 /-1.00$ \\
\hline FMINSRF2 & 1024 & $276 / 558 / 282 / 0.21$ & $245 / 491 / 246 / 0.19$ & $257 / 517 / 517 / 0.30$ \\
\hline SPMSRTLS & 1000 & $142 / 291 / 151 / 0.15$ & $135 / 277 / 144 / 0.14$ & $138 / 281 / 281 / 0.20$ \\
\hline LIARWHD & 5000 & $21 / 48 / 32 / 0.16$ & $20 / 42 / 27 / 0.15$ & $16 / 46 / 46 / 0.18$ \\
\hline NONDIA & 10000 & $9 / 22 / 16 / 0.24$ & $8 / 18 / 11 / 0.21$ & $6 / 26 / 26 / 0.28$ \\
\hline POWELLSG & 5000 & $162 / 332 / 187 / 0.27$ & $99 / 201 / 112 / 0.19$ & $148 / 346 / 346 / 0.34$ \\
\hline ARWHEAD & 10000 & $10 / 22 / 15 / 0.55$ & $8 / 25 / 21 / 0.58$ & $-1 /-1 /-1 /-1.00$ \\
\hline SROSENBR & 10000 & $12 / 26 / 17 / 0.16$ & $10 / 22 / 13 / 0.17$ & $8 / 26 / 26 / 0.20$ \\
\hline TQUARTIC & 5000 & $21 / 52 / 38 / 0.20$ & $16 / 47 / 38 / 0.20$ & $9 / 32 / 32 / 0.19$ \\
\hline PENALTY1 & 5000 & $47 / 106 / 62 / 0.17$ & $52 / 125 / 81 / 0.19$ & $38 / 152 / 152 / 0.25$ \\
\hline DQDRTIC & 10000 & $7 / 15 / 8 / 0.28$ & $7 / 15 / 8 / 0.28$ & $5 / 15 / 15 / 0.30$ \\
\hline NONDIA & 5000 & $8 / 27 / 22 / 0.14$ & $14 / 43 / 35 / 0.18$ & $5 / 26 / 26 / 0.14$ \\
\hline ARGLINB & 300 & $9 / 19 / 23 / 0.27$ & $12 / 20 / 27 / 0.27$ & $-1 /-1 /-1 /-1.00$ \\
\hline DIXMAAND & 6000 & $12 / 25 / 13 / 0.14$ & $11 / 23 / 12 / 0.12$ & $8 / 25 / 25 / 0.15$ \\
\hline ARGLINC & 300 & $8 / 16 / 19 / 0.26$ & $11 / 20 / 26 / 0.27$ & $-1 /-1 /-1 /-1.00$ \\
\hline DQRTIC & 5000 & $33 / 67 / 34 / 0.09$ & $49 / 99 / 50 / 0.11$ & $17 / 66 / 66 / 0.10$ \\
\hline QUARTC & 5000 & $33 / 67 / 34 / 0.09$ & $49 / 99 / 50 / 0.11$ & $17 / 66 / 66 / 0.09$ \\
\hline EIGENALS & 110 & $376 / 757 / 389 / 0.10$ & $391 / 787 / 405 / 0.10$ & $394 / 806 / 806 / 0.17$ \\
\hline SINQUAD & 500 & $59 / 122 / 111 / 0.03$ & $42 / 102 / 92 / 0.03$ & $-1 /-1 /-1 /-1.00$ \\
\hline SPARSINE & 200 & $444 / 889 / 445 / 0.07$ & $441 / 884 / 443 / 0.07$ & $457 / 917 / 917 / 0.10$ \\
\hline DIXON3DQ & 500 & $499 / 999 / 500 / 0.07$ & $499 / 999 / 500 / 0.06$ & $499 / 1003 / 1003 / 0.09$ \\
\hline DIXMAANC & 6000 & $10 / 21 / 11 / 0.12$ & $11 / 23 / 12 / 0.12$ & $7 / 23 / 23 / 0.17$ \\
\hline HILBERTB & 200 & $5 / 11 / 6 / 0.22$ & $5 / 11 / 6 / 0.22$ & $5 / 13 / 13 / 0.25$ \\
\hline BROWNAL & 400 & $4 / 15 / 13 / 0.21$ & $5 / 11 / 7 / 0.19$ & $4 / 37 / 37 / 0.26$ \\
\hline EIGENCLS & 90 & $358 / 718 / 360 / 0.07$ & $423 / 852 / 429 / 0.08$ & $366 / 743 / 743 / 0.13$ \\
\hline ARGLINA & 300 & $1 / 3 / 2 / 0.32$ & $1 / 3 / 2 / 0.28$ & $1 / 5 / 5 / 0.30$ \\
\hline EXTROSNB & 50 & $5108 / 10749 / 5819 / 0.24$ & $4325 / 9031 / 4848 / 0.17$ & $3574 / 7808 / 7808 / 0.23$ \\
\hline PENALTY2 & 200 & $199 / 234 / 365 / 0.14$ & $189 / 223 / 349 / 0.13$ & $-1 /-1 /-1 /-1.00$ \\
\hline FREUROTH & 1000 & $124 / 209 / 187 / 0.14$ & $54 / 99 / 92 / 0.08$ & $-1 /-1 /-1 /-1.00$ \\
\hline BRYBND & 1000 & $51 / 103 / 52 / 0.07$ & $32 / 65 / 34 / 0.05$ & $30 / 73 / 73 / 0.08$ \\
\hline DIXMAANB & 3000 & $9 / 19 / 10 / 0.06$ & $9 / 19 / 10 / 0.06$ & $6 / 23 / 23 / 0.07$ \\
\hline NONCVXU2 & 100 & $341 / 639 / 396 / 0.03$ & $382 / 680 / 484 / 0.04$ & $397 / 801 / 801 / 0.06$ \\
\hline DIXMAANA & 3000 & $9 / 19 / 10 / 0.06$ & $8 / 17 / 9 / 0.05$ & $7 / 20 / 20 / 0.07$ \\
\hline TOINTGSS & 10000 & $4 / 9 / 5 / 0.21$ & $4 / 9 / 5 / 0.19$ & $4 / 20 / 20 / 0.37$ \\
\hline POWER & 1000 & $116 / 233 / 117 / 0.03$ & $122 / 245 / 123 / 0.03$ & $114 / 236 / 236 / 0.03$ \\
\hline DECONVU & 61 & $457 / 916 / 462 / 0.06$ & $273 / 547 / 276 / 0.04$ & $281 / 581 / 581 / 0.05$ \\
\hline GENROSE & 100 & $305 / 641 / 347 / 0.02$ & $293 / 608 / 325 / 0.02$ & $298 / 626 / 626 / 0.02$ \\
\hline COSINE & 1000 & $12 / 28 / 24 / 0.03$ & $12 / 29 / 23 / 0.04$ & $9 / 29 / 29 / 0.03$ \\
\hline DIXMAANB & 1500 & $9 / 19 / 10 / 0.03$ & $9 / 19 / 10 / 0.03$ & $7 / 24 / 24 / 0.02$ \\
\hline CHNROSNB & 50 & $272 / 545 / 273 / 0.01$ & $242 / 487 / 245 / 0.01$ & $243 / 500 / 500 / 0.02$ \\
\hline DIXMAANA & 1500 & $9 / 19 / 10 / 0.03$ & $8 / 17 / 9 / 0.04$ & $7 / 22 / 22 / 0.03$ \\
\hline FMINSRF2 & 121 & $113 / 228 / 115 / 0.02$ & $115 / 232 / 117 / 0.02$ & $123 / 250 / 250 / 0.02$ \\
\hline ARWHEAD & 1000 & $10 / 24 / 16 / 0.03$ & $7 / 19 / 14 / 0.03$ & $-1 /-1 /-1 /-1.00$ \\
\hline
\end{tabular}

Table 2 - (continuation). 


\begin{tabular}{lllll}
\hline & & cg-descent & Algorithm 2.1 & prp + \\
\hline problem & $\mathrm{n}$ & iter $/ \mathrm{fn} / \mathrm{gn} /$ time & iter $/ \mathrm{fn} /$ gn $/$ time & iter $/ \mathrm{fn} /$ gn $/$ time \\
\hline COSINE & 500 & $12 / 29 / 23 / 0.01$ & $12 / 29 / 23 / 0.00$ & $9 / 26 / 26 / 0.00$ \\
DQDRTIC & 1000 & $7 / 15 / 8 / 0.03$ & $7 / 15 / 8 / 0.02$ & $5 / 15 / 15 / 0.03$ \\
ERRINROS & 50 & $1013 / 2023 / 1444 / 0.06$ & $1243 / 2432 / 1750 / 0.07$ & $-1 /-1 /-1 /-1.00$ \\
EG2 & 1000 & $4 / 9 / 6 / 0.02$ & $4 / 9 / 6 / 0.01$ & $-1 /-1 /-1 /-1.00$ \\
TESTQUAD & 100 & $297 / 595 / 321 / 0.01$ & $282 / 565 / 307 / 0.00$ & $425 / 925 / 925 / 0.02$ \\
TOINTGOR & 50 & $121 / 222 / 151 / 0.01$ & $121 / 221 / 152 / 0.01$ & $122 / 250 / 250 / 0.03$ \\
FMINSRF2 & 15625 & $514 / 1030 / 516 / 7.71$ & $492 / 985 / 493 / 6.58$ & $503 / 1012 / 1012 / 10.55$ \\
FMINSRF2 & 5625 & $369 / 741 / 372 / 2.69$ & $340 / 684 / 344 / 1.54$ & $350 / 707 / 707 / 2.47$ \\
NONDQUAR & 10000 & $10008 / 20026 / 10965 / 31.39$ & $4469 / 8958 / 4734 / 13.56$ & $10006 / 20100 / 20100 / 39.93$ \\
FMINSURF & 15625 & $734 / 1471 / 737 / 11.00$ & $706 / 1413 / 707 / 9.67$ & $734 / 1473 / 1473 / 15.90$ \\
FMINSURF & 10000 & $605 / 1213 / 608 / 4.92$ & $590 / 1182 / 592 / 4.75$ & $609 / 1223 / 1223 / 7.25$ \\
FREUROTH & 10000 & $90 / 157 / 142 / 1.27$ & $40 / 82 / 66 / 0.74$ & $-1 /-1 /-1 /-1.00$ \\
BDQRTIC & 10000 & $3085 / 6520 / 7298 / 42.44$ & $1879 / 4036 / 4982 / 27.90$ & $-1 /-1 /-1 /-1.00$ \\
NONCVXUN & 500 & $7123 / 12606 / 8771 / 3.57$ & $3009 / 5121 / 3912 / 1.60$ & $3386 / 6779 / 6779 / 2.40$ \\
GENROSE & 5000 & $11659 / 23403 / 11757 / 32.93$ & $10627 / 21303 / 10688 / 29.39$ & $10384 / 20823 / 20823 / 41.83$ \\
EIGENALS & 930 & $2920 / 5846 / 2930 / 20.76$ & $2704 / 5414 / 2715 / 18.80$ & $3069 / 6171 / 6171 / 36.16$ \\
GENHUMPS & 5000 & $6654 / 13394 / 6765 / 58.18$ & $6776 / 13803 / 7070 / 60.56$ & $7455 / 15193 / 15193 / 99.11$ \\
TESTQUAD & 10000 & $2231 / 4463 / 2232 / 5.28$ & $2234 / 4469 / 2235 / 4.91$ & $2045 / 4093 / 4093 / 6.41$ \\
TESTQUAD & 5000 & $1715 / 3431 / 1716 / 1.84$ & $1650 / 3301 / 1651 / 2.35$ & $1634 / 3271 / 3271 / 2.46$ \\
CHAINWOO & 1000 & $436 / 843 / 498 / 0.35$ & $284 / 560 / 355 / 0.25$ & $-1 /-1 /-1 /-1.00$ \\
\hline & & & & \\
\hline
\end{tabular}

Table 2 - (continuation).

\section{Numerical results}

In this section, we compare the performance of the proposed methods with those of the PRP+ method developed by Gilbert and Nocedal [10], and the CG_DESCENT method proposed by Hager and Zhang [11].

The PRP+ code was obtained from Nocedal's web page at http://www.ece. northwestern.edu/ ${ }^{\sim}$ nocedal/software.html, and the CG_DESCENT code from Hager's web page at http://www.math.ufl.edu/ ${ }^{\sim}$ hager/. The PRP+ code is coauthored by Liu, Nocedal and Waltz, and the CG_DESCENT code is coauthored by Hager and Zhang. The test problems are unconstrained problems in the CUTE library [3].

We stop the iteration if the inequality $\left\|g\left(x_{k}\right)\right\|_{\infty} \leq 10^{-6}$ is satisfied. All codes were written in Fortran and run on PC with $2.66 \mathrm{GHz}$ CPU processor and $1 \mathrm{~GB}$ RAM memory and Linux operation system. Tables 1 and 2 list all numerical results. For convenience, we give the meanings of these methods in the tables.

- "cg-descent" stands for the CG_DESCENT method with the approximate Wolfe line search [11]. Here we use the Source code Fortran 77 Version 1.4 (November 14, 2005) on Hager's web page and default parameters 
there;

- "Algorithm 2.1" is Algorithm 2.1 with $\rho=1$ and the same line search as "cg-descent";

- "prp+" means the PRP+ method with the strong Wolfe line search proposed by Moré and Thuente [16].

In order to get relatively better $\rho$ values in Algorithm 2.1, we choose 10 complex problems to test Algorithm 2.1 with different $\rho$ values. Table 1 lists these numerical results, where "problem", "iter", "fn", "gn", "time", “ $\|g(x)\|_{\infty}$ " and " $f(x)$ " mean the name of the test problem, the total number of iterations, the total number of function evaluations, the total number of gradient evaluations, the CPU time in seconds, the infinity norm of the final value of the gradient and the final value of the function at the final point, respectively.

In Table 1, we see that Algorithm 2.1 with $\rho=1$ performed best. Moreover, we also compared Algorithm 2.1 with other Algorithms in the previous sections and numerical results showed that they performed similarly. So in this section, we only listed the numerical results for Algorithm 2.1 with $\rho=1$, the "cgdescent" and "prp+" methods. These results are reported in Table 2 where "-1" means the method failed.

Figures 1-4 show the performance of the above methods relative to CPU time, the number of iterations, the number of function evaluations and the number of gradient evaluations, respectively, which were evaluated using the profiles of Dolan and Moré [7]. For example, the performance profiles with respect to CPU time means that for each method, we plot the fraction $\mathrm{P}$ of problems for which the method is within a factor $\tau$ of the best time. The left side of the figure gives the percentage of the test problems for which a method is the fastest; the right side gives the percentage of the test problems that are successfully solved by each of the methods. The top curve is the method that solved the most problems in a time that was within a factor $\tau$ of the best time.

Figure 1 shows that "Algorithm 2.1" performed slightly better than the "cgdescent" method did for the test problems. It outperforms the "cg-descent" and "prp+" methods for about 59\% (71 out of 120) test problems. "Algorithm 2.1" and the "cg-descent" method ultimately solve $100 \%$ of the test problems. The "prp+" method performed worst since it only solves $77 \%$ of the test problems 


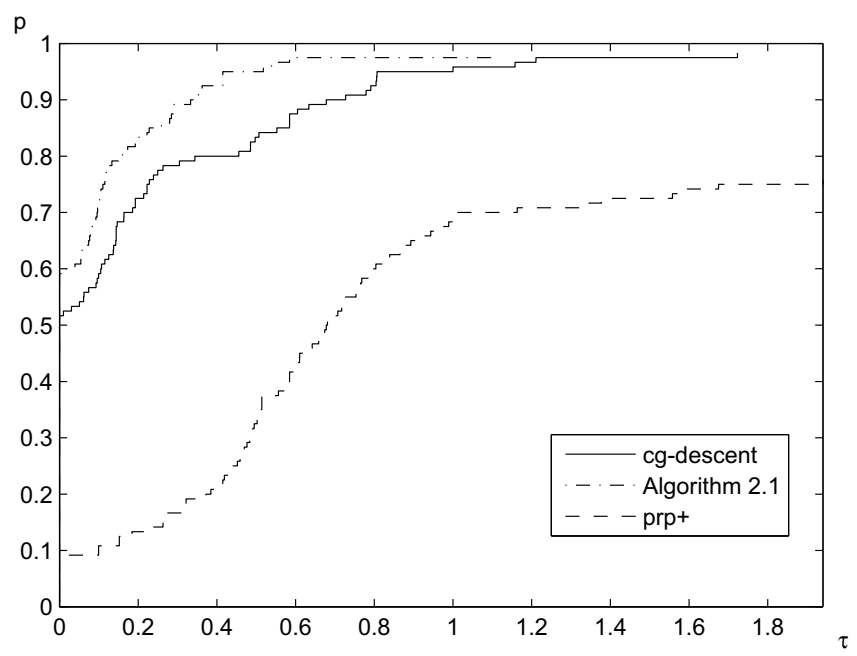

Figure 1 - Performance profiles with respect to CPU time.

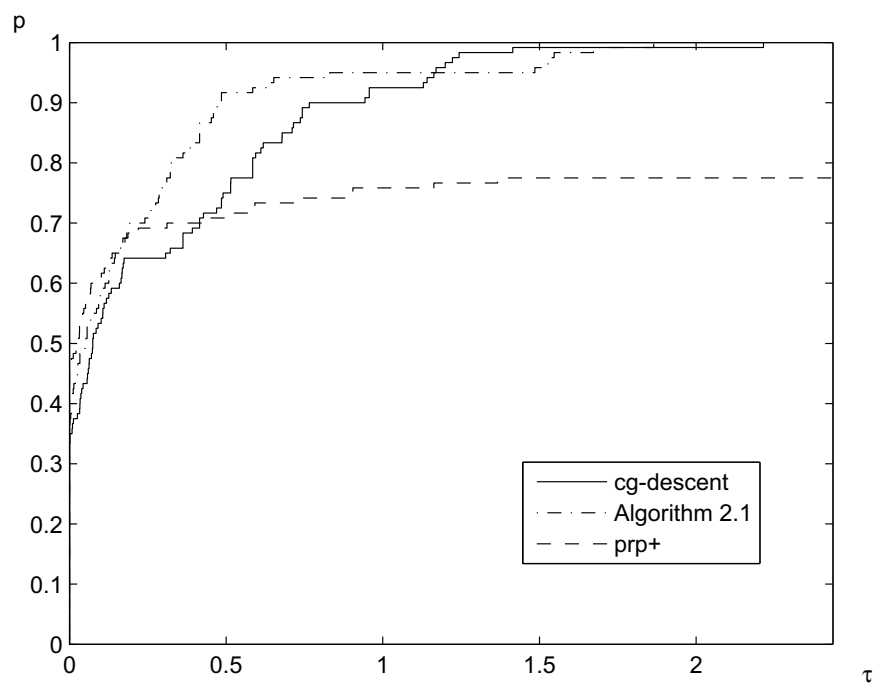

Figure 2 - Performance profiles with respect to the number of iterations.

successfully. But Figure 2 shows that "prp+" has the best performance with respect to the number of iterations since it solves about $46 \%$ of the problems with the smallest number of iterations. We can see from Figures 3 and 4 that "Algorithm 2.1" has the best performance with respect to the number of function and gradient evaluations since it corresponds to the top curve. 


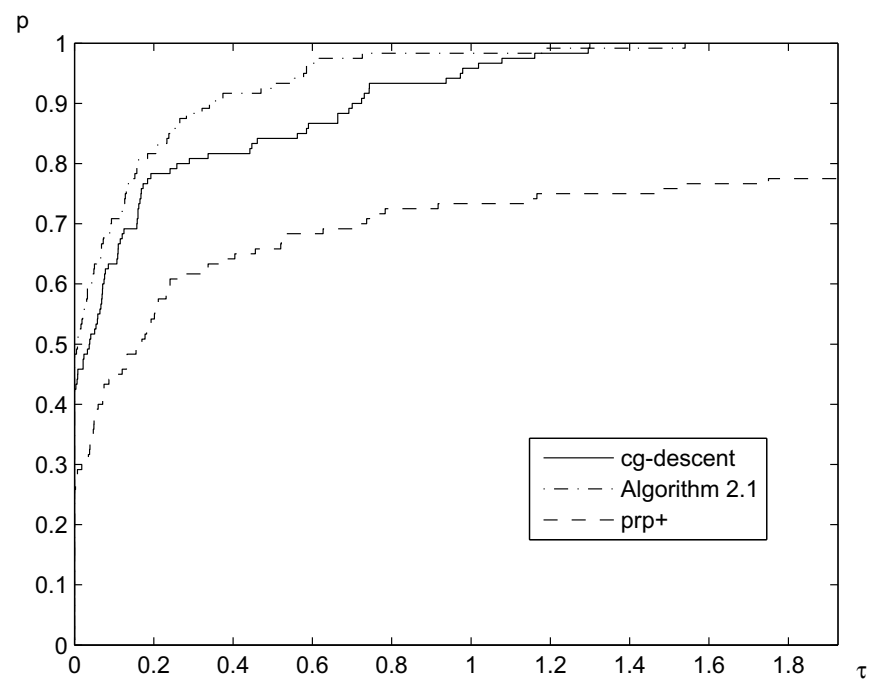

Figure 3 - Performance profiles with respect to the number of function evaluations.

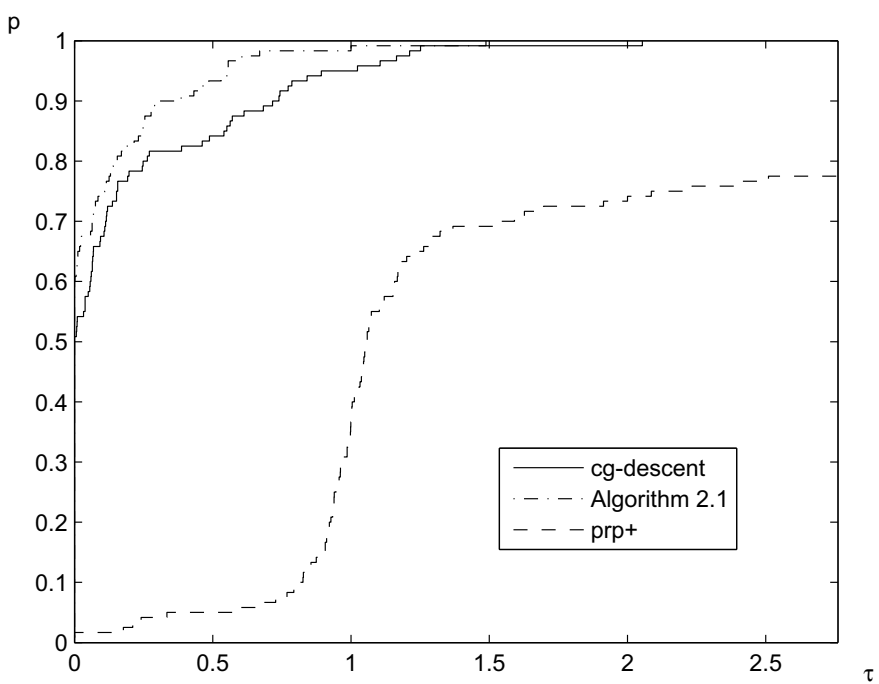

Figure 4 - Performance profiles with respect to the number of gradient evaluations.

\section{Conclusions}

We have proposed some new versions of the HS method based on the secant condition, which can generate sufficient descent directions with inexact line 
searches. Moreover, we proved that the proposed HS methods converge globally for strongly convex functions. Two modified schemes are introduced and proved to be globally convergent for general nonconvex functions. These results are also extended to some other conjugate gradient methods. Some results of the paper extend some work of the references [22, 23, 24]. The performance profiles showed that the proposed methods are also efficient for problems from the CUTE library.

Acknowledgement. This work was supported by the NSF foundation (10701018) of China.

\section{REFERENCES}

[1] N. Andrei, Scaled conjugate gradient algorithms for unconstrained optimization. Comput. Optim. Appl., 38 (2007), 401-416.

[2] E. Birgin and J.M. Martínez, A spectral conjugate gradient method for unconstrained optimization. Appl. Math. Optim., 43 (2001), 117-128.

[3] K.E. Bongartz, A.R. Conn, N.I.M. Gould and P.L. Toint, CUTE: constrained and unconstrained testing environments. ACM Trans. Math. Softw., 21 (1995), 123-160.

[4] Y.H. Dai and L.Z. Liao, New conjugate conditions and related nonlinear conjugate gradient methods. Appl. Math. Optim., 43 (2001), 87-101.

[5] Y.H. Dai and Y. Yuan, A nonlinear conjugate gradient method with a strong global convergence property. SIAM J. Optim., 10 (1999), 177-182.

[6] Y.H. Dai and Y. Yuan, Nonlinear Conjugate Gradient Methods. Shanghai Science and Technology Publisher, Shanghai (2000).

[7] E.D. Dolan and J.J. Moré, Benchmarking optimization software with performance profiles. Math. Program., 91 (2002), 201-213.

[8] R. Fletcher and C. Reeves, Function minimization by conjugate gradients. Comput. J., 7 (1964), 149-154.

[9] R. Fletcher, Practical Methods of Optimization, Vol I: Unconstrained Optimization. John Wiley \& Sons, New York (1987).

[10] J.C. Gilbert and J. Nocedal, Global convergence properties of conjugate gradient methods for optimization. SIAM. J. Optim., 2 (1992), 21-42.

[11] W.W. Hager and H. Zhang, A new conjugate gradient method with guaranteed descent and an efficient line search. SIAM J. Optim., 16 (2005), 170-192.

[12] W.W. Hager and H. Zhang, A survey of nonlinear conjugate gradient methods. Pacific J. Optim., 2 (2006), 35-58. 
[13] M.R. Hestenes and E.L. Stiefel, Methods of conjugate gradients for solving linear systems. J. Research Nat. Bur. Standards Section B, 49 (1952), 409-432.

[14] D. Li and M. Fukushima, A modified BFGS method and its global convergence in nonconvex minimization. J. Comput. Appl. Math., 129 (2001), 15-35.

[15] Y.L. Liu and C.S. Storey, Efficient generalized conjugate gradient algorithms, Part 1: Theory. J. Optim. Theory Appl., 69 (1991), 129-137.

[16] J.J. Moré and D.J. Thuente, Line search algorithms with guaranted sufficient decrease. ACM Trans. Math. Softw., 20 (1994), 286-307.

[17] B. Polak and G. Ribiere, Note sur la convergence des méthodes de directions conjuguées. Rev. Française Informat Recherche Operationelle, 16 (1969), 35-43.

[18] B.T. Polyak, The conjugate gradient method in extreme problems. USSR Comp. Math. Math. Phys., 9 (1969), 94-112.

[19] D.F. Shanno, Conjugate gradient methods with inexact searches. Math. Oper. Res., 3(1978), 244-256.

[20] P. Wolfe, Convergence conditions for ascent methods. SIAM Rev., 11 (1969), 226-235.

[21] Y. Yuan and J. Stoer, A subspace study on conjugate algorithms. ZAMM Z. Angew. Math. Mech., 75 (1995), 69-77.

[22] L. Zhang, W. Zhou and D. Li, A descent modified Polak-Ribière-Polyak conjugate gradient method and its global convergence. IMA J. Numer. Anal., 26 (2006), 629-640.

[23] L. Zhang, W. Zhou and D. Li, Global convergence of a modified Fletcher-Reeves conjugate gradient method with Armijo-type line search. Numer. Math., 104 (2006), 561-572.

[24] L. Zhang, W. Zhou and D. Li, Some descent three-term conjugate gradient methods and their global convergence. Optim. Methods Softw., 22 (2007), 697-711.

[25] G. Zoutendijk, Nonlinear Programming, Computational methods, Integer and Nonlinear Programming. J.Abadie (ed.), North-Holland, Amsterdam (1970), 37-86. 